\title{
Interfering with leukocyte trafficking in Crohn's Disease
}

\author{
Sujata Biswas ${ }^{1}$, Robert V Bryant ${ }^{2}$, Simon Travis ${ }^{1}$ \\ 1 Translational Gastroenterology Unit, NIHR Oxford Biomedical Research Centre, Oxford \\ University Hospitals NHS Foundation Trust, John Radcliffe Hospital, Oxford OX3 9DU, UK \\ sujata.biswas@ouh.nhs.uk \\ simon.travis@ndm.ox.ac.uk \\ robert.bryant@sa.gov.au
}

${ }^{2}$ IBD Service, Department of Gastroenterology, The Queen Elizabeth Hospital, Woodville South, Adelaide, South Australia, 5011, Australia.

\section{Abstract $(150$ words max)}

The discovery of gut-specific leukocytes and the ability to modulate their function has been a groundbreaking development in the treatment of inflammatory bowel disease. Drugs target the interaction between lymphocytes and endothelial cells via integrins and their ligand cellular adhesion molecules. Safety, efficacy and sustainability of effect has become the measure of this drug class, notwithstanding the association of natalizumab with fatal polyoma virus infection. Vedolizumab (2014) now licensed for the treatment of Crohn's disease around the world provides gut-specific immunosuppression. Targets for modulators of leukocyte trafficking include (examples in brackets) ICAM-1 (alicaforsen, efalizumab); MAdCAM-1(PF-00547659); 74 and related receptors (abrilumab, etrolizumab, natalizumab, vedolizumab); chemokine receptor CCR9 (vercirnon); and sphingosine 1-phosphate receptors (etrasimod, fingolimod, ozanimod). Oral and subcutaneous therapies are in development. The efficacy, safety and practice points of licensed drugs are discussed, in addition to initial results from therapeutic trials.

\section{Introduction}

A therapeutic revolution in the treatment of Crohn's disease (CD) started with the discovery that dysregulated, organ-specific lymphocytes are recruited to sites of inflammation (1). Surface molecules on gut-homing lymphocytes were identified and drugs designed to target the interaction between these molecules and gut endothelial cells. Safety issues plagued the early development of therapies that interfered with lymphocyte trafficking, notably natalizumab that was associated with progressive multifocal leukoencephalopathy (PML). Paradoxically, targeting gut-specific $\alpha 4 \beta 7$ integrins with vedolizumab has become a byword for safety. Nevertheless, the subset of immune cells which traffic to the gut is not entirely understood, nor are the reasons that response to leukocyte-directed therapies differs between phenotypes.

This review discusses the mechanisms of action of drugs that modify leukocyte trafficking, outline the efficacy and sustainability of therapy, focus on safety and provide practical guidance on therapeutic strategies in Crohn's disease. 


\section{$\underline{\text { 2. Mechanisms of leukocyte trafficking in Crohn's disease }}$}

\section{Leukocyte trafficking}

Recruitment and retention of leukocytes in the gut is a hallmark of chronic intestinal inflammation and a pivotal pathway in the immunopathogenesis of Crohn's disease.

The gut is one of the largest reservoirs of immune cells in the human body, both within the intestinal epithelium and in GALT (gut-associated lymphoid tissue). Rapid recruitment and retention of leukocytes is a hallmark of chronic inflammation. Leukocyte trafficking is, therefore, a highly regulated process. Complex interactions between circulating leukocytes and intestinal vascular endothelial cells allow managed migration of leukocytes across endothelium and into the intestinal mucosa (2). Migration is controlled by the orchestrated expression and regulation of adhesion molecules, chemokines and chemokine receptors, present both in stromal and in immune cells. Circulating immune cells are captured, roll, undergo activation, firmly adhere, and finally migrate into damaged tissue. Selectins on the surface of intestinal endothelial cells act as a foot brake to slow the flow of circulating leukocytes; selectins form low-affinity bonds with glycoproteins on the leukocytes and allow the cell to begin to adhere to the endothelium. Integrins and corresponding adhesion molecules apply the hand brake: full adhesion is mediated by the stable binding of integrin receptor molecules on the leukocyte to inducible cell adhesion molecule ligands on the surface of the intestinal endothelial cell. Within the context of inflammation, chemokines are induced on endothelial cells, which act as chemoattractants for their receptors on rolling leukocytes to promote their activation and subsequent migration across the endothelium (3). Drugs target all the different mediators of this process: cell adhesion ligands, integrin receptors and chemokines (figure 1).

Figure 1: Leukocyte signalling and targets for drug therapy. Leukocyte adhesion is mediated by integrins on leukocytes interacting with cellular adhesion molecules on endothelial cells. ICAM-1: intercellular adhesion molecule-1, MAdCAM-1: mucosal addressin cellular adhesion molecule-1, VCAM-1: vascular cell adhesion molecule-1.

\section{Integrin-ligand signaling}

Integrins are heterodimeric receptors expressed on the surface of circulating leukocytes, composed of $\alpha$ and $\beta$ subunits. Each subunit is a transmembrane protein with a small cytoplasmic domain and a much larger extracellular domain. Several forms of $\alpha$ and $\beta$ subunits exist, which make up different combinations of integrin molecules expressed on human leukocytes. These combinations can be specific to a particular type of leukocyte, or determine the target tissue for leukocyte trafficking. For example, CD11a (LFA-1) is specific to T-lymphocytes, while $\alpha 4 \beta 7$ is a marker for leukocyte trafficking specifically to the intestine. The terms 'ligand' and even 'receptor' are potentially confusing. They are best thought of as 'key' (ligand) and 'lock' (receptor). Chemokines provide part of the signaling that matches the two, in a rather more sophisticated fashion than colour-coding of keys and locks. Confusion can also arise because the interaction is two-way; the ligand for a receptor is in turn the receptor for its ligand. We will endeavor to be consistent in using the term 'receptor' for the molecule on leukocytes and 'ligand' for that on endothelial cells.

The natural ligands for integrin receptors are cellular adhesion molecules (CAMs). CAMs are members of the immunoglobulin superfamily that are expressed on the surface of vascular endothelial cells. Three CAMs in particular are known to play a role in leukocyte 
trafficking to the inflamed intestine: intracellular adhesion molecule (ICAM)-1, mucosal addressin cellular adhesion molecule (MadCAM)-1, and vascular cell adhesion molecule (VCAM)-1. ICAM-1 is constitutively expressed at low levels on the surface of intestinal endothelial cells and is induced by exposure to pro-inflammatory cytokines during inflammation. ICAM-1 allows migration of the leukocyte into the inflamed mucosa by binding to its receptor, the CD11a/CD18 integrin ( $\alpha \mathrm{L} \beta 2$, or LFA-1) on circulating leukocytes. MAdCAM-1 is expressed on vascular endothelial cells within the Peyer's patches and intestinal lymphoid tissues and binds to the $\alpha 4 \beta 7$ integrin receptor to regulate specific homing of memory/effector T cells to the intestine. VCAM-1 is transiently expressed on exposure to cytokines, and binds to one of its two receptors, $\alpha 4 \beta 1$ or $\alpha 4 \beta 7$. The $\alpha 4 \beta 1$ integrin largely mediates leukocyte trafficking to the central nervous system, while $\alpha 4 \beta 7$ mediates homing to the intestine. Consequently an inhibitor of $\alpha 4$ (such as natalizumab) blocks both pathways, while an inhibitor of $\alpha 4 \beta 7$ (such as vedolizumab) only blocks leukocyte traffic to the gut. Just how specific to the gut $\alpha 4 \beta 7$ expression is in all circumstances remains to be determined, but at present it appears to be specific for clinical purposes. 77 also associates with other subunits, including $\alpha E$, which is expressed in lung tissue as well as the gut. There is a role for CAMs in the pathogenesis of CD, making blockade of the integrin-ligand pathway an appealing strategy for drug development.

\section{Animal models}

Animal models of IBD have been instrumental in testing modifiers of leukocyte function (4). DSS and TNBS models of chemical colitis were used to show that selectin inhibition had no effect on disease (5), but that targeting integrins and their receptors did. Scientists should remember that this is an unusual example of murine models translating into clinical practice, especially in Crohn's disease! Anti-ICAM-1 antibody attenuated colitis and there was prolonged survival in DSS-treated ICAM-1 deficient mice (6) (7). When antiVCAM-1, anti-MAdCAM-1 and anti-ICAM-1 were compared, anti-VCAM-1 proved superior in both DSS and TNBS models (8). In an experimental model of spontaneous ileitis, concomitant ICAM-1 and VCAM-1 blockade showed therapeutic effect (9).

Animal studies also demonstrated that $\alpha 4$ integrin blockade, which inhibits both $\alpha 4 \beta 1$, the integrin receptor for VCAM-1, and $\alpha 4 \beta 7$, the gut-specific integrin receptor for MAdCAM-1, attenuated colitis (10). Murine models supported the relevance of the $\alpha 4 \beta 7$ axis in intestinal inflammation, since antibodies against $\beta 7$ or MAdCAM-1 reduced colonic inflammation (11).

\section{Drug development in leukocyte trafficking}


Table 1: Drugs, mechanistic target and phase of development

\begin{tabular}{|l|l|l|}
\hline Drug & Target & Development phase \\
\hline Alicaforsen & ICAM-1 & Phase II ; negative (12) \\
\hline Efalizumab & ICAM-1/LFA-1 & $\begin{array}{l}\text { Pilot trial (13) } \\
\text { Withdrawn before further testing }\end{array}$ \\
\hline PF-00547659 & MAdCAM-1 & Phase II ; negative (14) \\
\hline Natalizumab & $\alpha 4 \beta 1$ and $\alpha 4 \beta 7$ & Approved then withdrawn (15, 16) \\
\hline Vedolizumab & $\alpha 4 \beta 7$ & Approved (17) \\
\hline Abrilumab & $\alpha 4 \beta 7$ & Phase II ; negative (18) \\
\hline Etrolizumab & $\beta 7$ & Phase III trial in progress \\
\hline CCX282 & CCR9 & Phase III ; negative (19, 20) \\
\hline Ozanimod & S1P1 and S1P5 & Phase II trial in progress \\
\hline Etrasimod & S1P1, S1P4, and S1P5 & Phase II trial planned \\
\hline
\end{tabular}

\section{a. ICAM-1/LFA-1 targeting}

ICAM-1 was the first intestinal CAM to be investigated as a target for therapeutic intervention in IBD. ICAM-1 expression was shown to be higher in areas of active inflammation in patients with IBD (21).

Alicaforsen is an antisense oligonucleotide which hybridises to a sequence within ICAM-1 and cleaves the heterodimer, leading to reduced expression of ICAM-1. An initial pilot study showed benefit in Crohn's disease but this was not confirmed in a randomised controlled trial in 2007 (12) (see table 1).

Efalizumab inhibits the binding of ICAM-1 to its receptor LFA-1 on the surface of circulating lymphocytes, via binding to CD11a. A small open-label study evaluated the efficacy and safety of efalizumab in 15 patients with moderate to severely active CD (13). By 8 weeks of treatment, 10/15 patients achieved clinical response (67\%), and 6/15 patients achieved clinical remission (40\%). No serious adverse events were reported. Despite this promising early result, efalizumab was voluntarily withdrawn from the US market in 2009 after three reported cases of PML in patients treated for psoriasis. Analysis of peripheral blood and cerebrospinal fluid revealed that both patients had reduced transendothelial migration of lymphocytes in vitro and decreased activation of CD8+ T cells. This suggests that LFA-1 plays a critical role in control of John Cunningham polyoma (JC) virus and that inhibition impacts recruitment of leukocytes to the central nervous system, where they are important for maintaining homeostatic control over the virus. This is relevant to all modulators of leukocyte trafficking, notably natalizumab. Until 
the molecular mechanisms are fully understood, all drugs in this class can be expected to be challenged on this issue.

\section{b. MAdCAM-1 targeting}

MAdCAM-1 is constitutively expressed and is upregulated in CD (22). PF-00547659 is an anti-MAdCAM-1 monoclonal antibody (23). Although a phase II study showed efficacy for induction of clinical remission at week 12 in UC, in CD the primary endpoint, defined as a response at week 8 or 12, was not met (24) (14). The drug was pharmacologically active, because there was a demonstrable dose-related reduction in soluble MAdCAM-1. The functional result of targeting the leukocyte integrin $\alpha 4 \beta 7$ or its ligand MAdCAM- 1 would be expected to be similar, but perhaps not the same. Binding an epitope expressed on leukocytes or on endothelial cells may have different effects, such as on antibodydependent cytotoxicity or induction of apoptosis.

\section{c. $\alpha 4 \beta 7$ targeting}

Natalizumab inhibits the biological activity of both $\alpha 4 \beta 1$ and $\alpha 4 \beta 7$. In 2003 a phase II clinical trial conducted in 248 patients with moderate to severely active CD showed that patients randomised to receive two infusions of either $3 \mathrm{mg} / \mathrm{kg}$ or $6 \mathrm{mg} / \mathrm{kg}$ of natalizumab had higher rates of clinical response and remission at multiple timepoints compared to placebo-treated patients, with similar rates of adverse events occurring between the groups (25).

In 2005, after FDA approval in multiple sclerosis (MS), two phase III studies in CD reported the efficacy of natalizumab for induction of remission (ENACT-1), as well as maintenance of response or remission (ENACT-2) (16). In ENACT-1, 948 patients with moderate to severely active $\mathrm{CD}$ were randomised to receive $3 \mathrm{mg} / \mathrm{kg}$ natalizumab or placebo at 0,4 , and 8 weeks; the primary endpoint was a 70-point reduction in the Crohn's Disease Activity Index (CDAI) at 10 weeks. In ENACT-2, 339 patients who initially responded to natalizumab during ENACT-1 were re-randomised to receive $3 \mathrm{mg} / \mathrm{kg}$ natalizumab or placebo every 4 weeks through week 56; the primary endpoint was the percentage of patients with a sustained clinical response at week 36 . The results showed that patients treated with natalizumab had a similar clinical response at week 10 compared to placebo ( $56 \%$ vs $49 \%, P=0.05$ ). Continuing natalizumab as maintenance therapy among responders led to high response rates compared to placebo (61 vs 28\%: $P<0.001$ ) at week 36 .

However, during an open-label extension of ENACT-2 one patient treated with natalizumab died of JC virus-associated PML. Natalizumab was removed from the market in 2005, but based on the results of the ENACT and ENCORE trials (15), natalizumab was approved by the FDA in 2008 for use as monotherapy in the treatment of moderate to severely active CD. Approval required strict post marketing surveillance under the TOUCH programme for education and monitoring for PML.

Vedolizumab is a humanised monoclonal antibody, approved (2014) for the treatment of both moderate to severely active CD and UC. Unlike natalizumab, vedolizumab targets the entire $\alpha 4 \beta 7$ integrin, which in theory should eliminate any associated risk of PML since $\alpha 4 \beta 7$ is specifically expressed on $\mathrm{T}$ cells that home to the intestine. 
The phase III trial (GEMINI II) in patients with moderate to severely active CD (17) had two primary endpoints for the induction phase, defined as clinical remission (CDAI < 150) and clinical response (100-point decrease in CDAI) at week 6. The primary endpoint for the maintenance phase was clinical remission at 52 weeks. During the induction phase, $14.5 \%$ of vedolizumab-treated patients achieved clinical remission by week 6 , compared to $6.8 \%$ of patients given placebo $(\mathrm{p}=0.02)$; no difference was observed in clinical response rates (31.4\% vs. $25.7 \%$ ). Among responders, $39.0 \%$ and $36.4 \%$ of patients maintained on vedolizumab every 8 and 4 weeks, respectively, were in remission at 52 weeks, compared to $21.6 \%$ of patients maintained on placebo (both $\mathrm{p}<0.01$ ) (see section $4 a)$.

Abrilumab is a newer anti- $\alpha 4 \beta 7$ antibody, which did not meet the primary endpoint of clinical remission at week 8 in two recent studies in either CD (18) or UC (26). However, higher rates of remission and response in patients with CD were observed in the active treatment arms at week 12 (41.7\% remission at $21 \mathrm{mg}$ dose vs $17.6 \%$ in placebo, $\mathrm{p}<0.1)$. Slow onset of action of anti- $\alpha 4 \beta 7$ antibodies in CD may be due to transmural inflammatory infiltrates showing resistance to apoptosis, leading to slower resolution of active inflammation. On the other hand, inhibition of leukocyte recruitment acts at an early stage of the inflammatory response, so can reasonably be expected to achieve sustainable remission by preventing recurrent inflammation.

Etrolizumab in a humanised anti- $\beta 7 \mathrm{mAb}$ that blocks intestinal-specific lymphocyte recruitment via $\alpha 4 \beta 7 / \mathrm{MAdCAM}-1$, as well the interaction of $\alpha \mathrm{E} \beta 7$ and its natural ligand, Ecadherin. A phase II trial in UC (27) was notable because remission could be predicted by higher levels of $\alpha \mathrm{E}$ in biopsies. Etrolizumab is now being tested in BERGAMOT, a phase III trial in CD. The proof of concept induction cohort of BERGAMOT in Crohn's compared $105 \mathrm{mg} 4$-weekly or $210 \mathrm{mg}$ at weeks $0,2,4,8$ and 12 weeks (28). At week 14 there was an improvement in symptoms and endoscopic score. Symptomatic remission at week 14 was $11.9 \%$ in placebo vs $20.8 \%$ in etrolizumab $105 \mathrm{mg}$ vs $24.8 \%$ in etrolizumab $210 \mathrm{mg}$. Results of BERGAMOT are expected in 2019 ;its extension study JUNIPER continues the herbal theme.

\section{d. Chemokine and chemokine receptor complex targeting}

CCR9 is a chemokine receptor expressed on the cell surface of memory/effector T cells that selectively binds to the potent small intestinal lymphocyte chemoattractant CCL25. The interaction of CCL25/CCR9 promotes adherence and migration of T lymphocytes and $\operatorname{IgA}$ antibody secreting cells into the small intestine.

Vercirnon (CCX282) is an orally active small molecule CCR9 antagonist that acts as a selective and potent inhibitor of CCL25-mediated lymphocyte chemotaxis. Although a phase II double-blind, randomised, placebo-controlled trial (PROTECT-1) did not achieve its primary endpoint of clinical response at 8 weeks, the study was interpreted positively since a clinically significant treatment effect was observed by 12 weeks (61\% response rate in vercirnon-treated patients vs. $47 \%$ with placebo) (19). Drug development proceeded with four planned phase III multicentre randomised controlled trials. However, in August 2013 the manufacturers announced that the first of four phase III trials (SHIELD-1) in patients with moderate to severely active CD also did not achieve its primary endpoint of clinical response at 12 weeks. There was also a dose-dependent increase in the frequency of adverse events. A further phase III trial failed to demonstrate 
efficacy as induction therapy (treatment difference $2.5 \%(\mathrm{p}=0.55)$ for once daily and $2.1 \%$ $(p=0.65)$ for twice daily) and maintenance was not addressed (20). Since CCL25 is predominantly expressed in the small intestine, it is conceivable that this attractive treatment target may yet be relevant to patients with isolated small intestinal CD.

\section{e. Targeting leukocyte circulation outside the intestine}

Blockade of lymphocyte recirculation in lymph nodes can be targeted through modulation of sphingosine 1-phosphate (S1P) receptors. Sphingosine 1-phosphate is a bioactive lysophospholipid metabolite that can act as an intercellular lipid mediator of inflammation. Interaction between S1P and the five known S1P receptors (1-5) regulate the response and function of biological processes, including cell differentiation, migration, proliferation and trafficking of $\mathrm{T}$ and $\mathrm{B}$ cells.

Fingolimod was the first S1P receptor modulator to reach the market, initially approved for the treatment of MS (29). The drug is phosphorylated in vivo, acquiring a structure similar to that of S1P, and functions as a non-selective small-molecule agonist to four of the five S1P receptors. Fingolimod causes peripheral blood lymphopenia due to sequestration of lymphocytes within lymphoid tissue and is associated with cardiovascular side effects, such as bradycardia and hypotension. This led to the development of more specific S1P receptor modulators with preferential actions on S1PR1, S1PR4 and S1PR5, since fingolimod also showed beneficial effects in murine models of colitis (30).

Ozanimod (RPC-1063) is an oral agonist, primarily of S1P1 and to a lesser extent of S1P5. The drug was also initially tested in MS: a phase II trial met the primary endpoint, showing a reduction in gadolinimum-enhancing lesions over a period of 24 weeks and has now progressed to phase III studies. In a double-blind phase II clinical trial in patients with active UC (2016), ozanimod at a dose of 0.5 or $1 \mathrm{mg}$ daily was administered for 32 weeks (31). The primary outcome, defined as clinical remission at 8 weeks, was higher in patients treated with ozanimod $1 \mathrm{mg}$ daily $(16.4 \%)$ than placebo $(6.2 \%, \mathrm{p}=0.048)$. Treatment with ozanimod was also associated with higher rates of clinical response and mucosal healing (34.3\% vs $12.3 \%$, $1 \mathrm{mg}$ vs placebo respectively) at week 8 , and higher rates of remission at week $32(26.2 \%$ vs $6.2 \%, 0.5 \mathrm{mg}$ daily vs placebo, $\mathrm{p}=0.002)$. A dosedependent decline in peripheral lymphocyte counts was observed. A phase II trial is underway in $\mathrm{CD}$.

Etrasimod (APD334) is an oral, next-generation S1P modulator that selectively targets S1P1, S1P4, and S1P5 in in vitro signalling assays. Preliminary results from the Phase II trial (OASIS) of etrasimod in 156 patients with moderate- to severely active UC have been presented, but not yet in CD (32). Exploratory endpoint results are most comparable to other studies, with clinical remission in $33.0 \%$ given $2 \mathrm{mg}$ etrasimod and $8.1 \%$ on placebo $(p<0.001)$ and endoscopic improvement in $41.8 \%$ and $17.8 \%$, respectively $(p=0.003)$. It seems to work faster, with less first dose-related bradycardia than ozanimod.

\section{Interfering with leukocyte trafficking in clinical practice: vedolizumab}

Of the drugs discussed above, only vedolizumab has entered widespread clinical practice for $\mathrm{CD}$, so its efficacy, safety, and prescribing in pregnancy, the elderly and perioperative period merit scrutiny.

\section{a. Efficacy}


The efficacy of vedolizumab in CD superficially appears less convincing than in UC. The drug failed to meet the primary endpoint in one of two induction trials (GEMINI II), but the timing ( 6 weeks) was unreasonably short for the mechanism of action. It was clearly effective by 10 weeks $(29 \%$ vs $13 \%$ on placebo, $\mathrm{p}=0.001)$. The effect size doubled by week 10 compared to week 6 in all patients, whether anti-TNF exposed or not.

Compared to anti-TNF therapy, vedolizumab appears to have a slower onset of action in CD. Nevertheless, symptomatic improvement in stool frequency is significantly greater than placebo within 2 weeks. Slower onset of action is not synonymous with reduced potency, since vedolizumab has high rates of remission and mucosal healing over 26 weeks. Patience is required in managing the expectations of patients, no different to using thiopurines. Some patients with particularly active disease may benefit from bridging treatment with steroids, which also enhance the effect of vedolizumab (clinical response at week 10 in $30.9 \%$ in patients on steroids in GEMINI III, compared to $16.5 \%$ on vedolizumab alone).

\section{$\underline{b .}$ Side effects}

Integrated data from registration trials included 2830 vedolizumab-exposed patients (4811 patient-years' exposure, PYs) and 513 placebo patients (33) Higher, but statistically insignificant rates of infection occurred on vedolizumab therapy (7.4/100 PYs; $95 \% \mathrm{CI}$ : 6.6-8.3) compared to placebo (6.7 PYs; 95\% CI: 3.2-10.1). Lower exposure-adjusted incidence rates of infection were reported after vedolizumab (63.5/100 PYs; 95\% CI: 59.6-67.3) and serious adverse events (20.0/100 PYs; 95\% CI: 18.5-21.5) compared to placebo (82.9/100 PYs; 95\% CI: 68.3-97.5) and (28.3/100 PYs 95\% CI: 20.6-35.9) respectively. One case of hypersensitivity during infusion was reported in a patient with CD.

The efficacy and safety of subcutaneous vedolizumab is currently being tested in a phase III trial (NCT02611817). For anti-TNF the mode of administration was similar in terms of efficacy and safety when subcutaneous adalimumab and certolizumab pegol were compared with intravenous infliximab (34). For the right patient subcutaneous administration gives the advantage of self-administration and fewer trips to hospital.

The GEMINI III study suggested that vedolizumab is appropriate for the young, elderly, patients with previous malignancy and those at risk of sepsis. Having been introduced into practice four years ago, PML has been reported during vedolizumab on a single occasion, in a patient with undiagnosed HIV infection with a CD 4 count $<400$ and after extended treatment with azathioprine (35) (33). It is difficult to believe that this was a consequence of vedolizumab.

\section{c. Infection}

Vedolizumab therapy was not associated with an increased incidence of respiratory tract infections compared with placebo (36). In the randomised controlled trials $(n=1731$ patients), the incidence of upper respiratory tract infections was numerically higher in 84 patients receiving vedolizumab compared with those on placebo, although this difference was not statistically significant (38.7 vs 33.0 patients per 100 PYs; hazard ratio 1.12; 95\% confidence interval: $0.83-1.51 ; \mathrm{p}=0.463)$. The rate of lower respiratory tract infections (LRTI), including pneumonia, was numerically lower in the vedolizumab versus the placebo group, but not statistically significant (7.7 vs 8.5 per 100 PYs (36)). Both URTIs and LRTIs were more frequent in patients with CD compared with UC, partly due to a 
higher rate of smoking in CD. Most RTIs in patients receiving vedolizumab were not serious and did not require treatment discontinuation. In terms of opportunistic infections vedolizumab has a favourable safety profile (37). Safety data from six trials were collated including 4811 patient years of vedolizumab exposure. Serious infections, Clostridium Difficile and TB were reported in $\leq 0.6 \%$ patients. Independent risk factors for serious infections in CD were younger age, steroid use or use of narcotic analgesia. Overall the safety data of vedolizumab in comparison to anti-TNF are reassuring.

There are no data on the secondary transmission of infection by live vaccines in patients receiving vedolizumab, so advice remains as for anti-TNF treatment: live vaccines are best avoided.

\section{d. Malignancy}

Relative to the general population, patients with $\mathrm{CD}$ are at a greater risk of gastrointestinal (GI) cancers. It has been estimated that in patients with $\mathrm{CD}$ the risk is increased 1.9-2.5-fold for colorectal cancer, 2.9-fold for upper GI and 27.1-fold for small bowel cancer. Furthermore, some treatments for IBD have been associated with an increased risk of malignancy, notably thiopurines, which were found in a meta-analysis to increase the incidence of lymphoma in patients with IBD by 4.9-fold (38). An increased risk of melanoma has also been seen with the use of biologics (39)(40), and the risk of non-melanoma skin cancer is increased with the use of thiopurines (41). A pooled analysis revealed a 1.8-fold greater incidence of malignancy in patients with $C D$ receiving immunomodulator therapy compared with those with no immunomodulator use.

Patients with a previous malignancy were excluded from clinical trials for vedolizumab, so it is not clear whether recurrence of malignancy is increased due to reduced immune (especially GI) surveillance. In the GEMINI studies covering 7694 patient-years of vedolizumab exposure, 28 patients developed malignant neoplasms, corresponding to less than $1 \%$ of all patients reporting AEs $(n=16577) .1 \%$ of patients enrolled in the open label extension developed a malignancy (42). In the post-marketing setting, 154 patients reported malignancies in 114071 patient-years (approximately 135 per 100000 patient-years), which was substantially lower than those reported in those in the open label extension study and in another study by Bernstein (43). Post-marketing data can be difficult to interpret, owing to difficulty in finding a suitable reference range for patients with IBD. Optum's Clinformatics Data Mart database (CDMD), a deidentified claims database, allows comparison of patients with IBD treated with vedolizumab in the Takeda long term safety study (LTSS) and global safety database (GSD) with patients with similar comorbidity. Of 2243 patients followed up for 7746 patient-years in the LTSS, 31 experienced a malignancy (17 CD, $14 \mathrm{UC}$ ); this was fewer than expected from the CDMD (31 vs 62; ratio: $0.50, \mathrm{p}<0.0001)$. The most common malignancies were renal and bladder (6/31) and lower GI (5/31). Prior anti-TNF use was reported in $61 \%$ patients and concomitant immunomodulators in $39 \%$. In the postmarketing GSD, 299 malignancies were reported in 293 patients in the context of 208 050 patient-years of exposure (including malignancies within 1 year of vedolizumab initiation). The number of observed malignancies in the LTSS was lower than expected from the CDMD. Numbers are small and should not be over-interpreted, as well as recognising the limitation that standardisation does not correct for other potential confounders (e.g. smoking and body mass index). All that can be said is that the number of malignancies with vedolizumab appeared low. 


\section{e. Immunogenicity}

Emerging pharmacokinetic data indicate that vedolizumab concentrations correlate with clinical outcomes, although the correlation may be weaker for vedolizumab than with anti-TNF agents. No link between drug concentration and individual patient response has been observed (44), although preliminary reports suggest that a level $>18 \mathrm{mcg} / \mathrm{mL}$ is associated with response (45).

Associations between concomitant use of immunomodulators and decreased immunogenicity of vedolizumab have been reported, but it does not make intuitive sense to convert gut-specific immunosuppression into systemic immunosuppression. Co-administration of azathioprine, mercaptopurine or methotrexate did not affect predicted vedolizumab clearance in a population pharmacokinetic-pharmacodynamic model (46). However, data are sparse and largely limited to post-hoc analyses.

Vedolizumab and anti-TNF drugs may differ in their immunogenicity. Among 620 patients with available blood samples in GEMINI 1, only $23(3.7 \%)$ were positive for anti-drug antibodies toward vedolizumab at any time, with $<1 \%$ showing persistence of anti-vedolizumab antibodies. In GEMINI II for CD, only $56 / 1434$ patients (4\%) had detectable anti-vedolizumab antibodies at any time during the 52 weeks of continuous treatment and these also failed to persist in most. These observations suggest that anti-vedolizumab antibodies are generated to a lesser extent than anti-TNF antibodies. However it is not certain that the anti-drug antibodies directed towards vedolizumab have the same functional consequence as those directed towards anti-TNF.

\section{f. Use in the elderly}

The risks of systemic immunosuppression are increased in the elderly. The trouble is that patients with IBD are getting older, so while only $12 \%$ of patients have IBD diagnosed over the age of 60 (the definition of elderly varies widely!), many patients with IBD starting at a younger age are now in their 70s and 80s, or older (47). Adverse events are more commonly reported in older patients. Postmarketing data identify events more commonly expected in this group, including pneumonia or death (48). Other AEs were reported at similar frequencies in both age groups. Most patients reporting AEs continued treatment. A single multicentre retrospective cohort study reported on vedolizumab initiated in patients over the age of 60 (mean 67.1 years) in 29 patients (10 UC, 19 CD) (48). Median treatment duration was 30 weeks. Three patients $(10 \%)$ experienced adverse events, including worsening of pneumonia, gastrointestinal symptoms, and flu-like symptoms. Limitations of post-marketing safety reports included voluntary reporting and difficulty establishing a causal relationship between the drug and event.

\section{g. Use in pregnancy}

Across six controlled trials, there were 27 pregnancies in female patients and 19 pregnancies in partners of male patients (49). Among 24 vedolizumabtreated females there were 11 live births, five elective terminations, four spontaneous abortions and four undocumented outcomes. A congenital corpus callosum agenesis anomaly was reported in one live birth from a healthy 
volunteer with extensive obstetric history exposed to singledose vedolizumab 79 days before estimated conception. Out of the 19 pregnancies in partners of male participants, there were 11 live births, two spontaneous abortions, three elective terminations and three undocumented outcomes. Post-marketing reports recorded 81 pregnancies resulting in four live births, 11 spontaneous abortions and 66 pregnancies that were on-going or reported undocumented outcomes.

The half-life of vedolizumab is 25 days, twice that of anti-TNF, so a strategy of withholding dosing during the third trimester would still theoretically result in a substantial concentration of vedolizumab in the fetus prior to delivery. This could lead to prolonged drug clearance in the newborn infant that could extend to 6-12 months. The implications are unclear. The global safety database (GSD) maintained by Takeda, holds post-marketing data reported by practitioners on 285 pregnancies in patients with CD related to vedolizumab. By the nature of voluntary post-marketing reports, data are incomplete, but 205/285 reported no adverse event. 29 spontaneous abortions were reported; other AEs included exacerbation of CD (23 reports), exposure of the partner (27) and off label use (45). The largest amount of monoclonal antibody is transferred across the placenta in the third trimester, whereas most of the $\mathrm{AE}$ reports relating to spontaneous abortion occurred in the first trimester. A low number of congenital abnormalities were reported, with insufficient information provided to assess causality in relation to vedolizumab treatment in most cases. No issues with fertility or fetal harm were observed in animal studies of vedolizumab .

It is not yet known whether vedolizumab is excreted in human milk, but as a protein digestion and inactivation can reasonably be anticipated in the neonatal gut. From a practical point of view, uncertainty about drug safety of vedolizumab during pregnancy is best discussed with patients with $\mathrm{CD}$ considering conception. Although safety cannot be confirmed, patients can be reassured that many patients have had normal pregnancies and that the benefits of breast feeding are likely to outweigh any risks.

\section{h. Peri-operative safety}

The GEMINI trials did not analyse surgical outcomes. Theoretically vedolizumab could impair wound healing by inhibiting the migration of leukocytes to sites of inflammation or repair in the intestine. There are three retrospective, singlecentre cohort studies on patients who had received at least one infusion of vedolizumab within 12 weeks of surgery. The largest cohort from the Mayo Clinic compared post-operative complications after vedolizumab exposure with biologic-naive and anti-TNF-exposed groups (50). Pre-operative treatment with vedolizumab was associated with an increased post-operative complication rate, most notably surgical site infection, although the anastomotic leak rate was similar across all groups. Multivariate analysis showed that vedolizumab increased the risk of surgical site infection 3-4 fold, although it is difficult to postulate a mechanism by which wound infection at the site of surgery could be increased by a drug that affects intestinal leukocyte trafficking without an increase in anastomotic leak rate. A smaller cohort study from California included 36 operations on 27 patients, reporting an overall complication rate of 
$53 \%$, infectious complications in $44 \%$ and anastomotic leak rate in $15 \%$ (51). Two deaths were reported, secondary to culture-negative sepsis. The third study from 15 patients undergoing intra-abdominal surgery reported just two postoperative complications (a stitch abscess and ileus requiring hospital readmission) (52). The data do not compare favourably to meta-analyses on perioperative anti-TNF therapy, which reported post-operative infectious complications in 15\%-17\% (53), but the vedolizumab studies were all small and retrospective, so further evidence is required before drawing conclusions.

\section{i. Extra-intestinal manifestations}

There are two studies evaluating vedolizumab in relation to extra-intestinal manifestations (EIMs) of IBD. In a French cohort (OBSERV-IBD), 173 patients with CD and 121 with UC treated with vedolizumab had activity of EIMs assessed at weeks 0, 6, 14, 22, 30 and 54. At baseline, 49/294 (17\%) patients had EIMs (47/49 inflammatory arthralgia/arthritis, 4/49 cutaneous lesions and 2/49 both). At week 54, 21/49 (45\%) had remission of arthritis and 75\% remission of skin lesions, but 34/245 (14\%) without EIM at baseline developed an EIM and 14/245 developed paradoxical skin manifestations. The GETAID authors conclude that EIMs may improve on VDZ, especially if IBD remission is achieved, but that EIMs may develop while on VDZ (54).

The second study used a different approach, by examining reports from a large US MarketScan claims' database, of EIMs that developed in all patients receiving vedolizumab compared to anti-TNF therapy (55). The incident rate ratio (IRR) for the development of any EIM was 1.28 (95\% CI 1.02-1.62), indicating a 28\% increased likelihood of an EIM developing on treatment with vedolizumab compared to anti-TNF therapy. The incidence rate ratio for specific EIMs was increase two-fold for episcleritis (IRR 2.51, 95\% CI 1.02-6.14), four-fold for pyoderma gangrenosum (IRR 4.42, 95\% CI 1.00-19.45)) and 7-fold for primary sclerosing cholangitis (IRR 7.79, 95\% CI 3.32-18.27). This suggests that patients receiving vedolizumab are more likely to develop EIMs than on anti-TNF therapy, but unlike OBSERV-IBD could not examine the impact of vedolizumab on EIMs.

\section{j. Perianal Crohn's}

The GEMINI II trial included 57 patients with draining fistulae at baseline. At 52 weeks, $41.2 \%$ of the VDZ 8-weekly group achieved fistula closure compared with $22.7 \%$ of the VDZ 4-weekly group and $11.1 \%$ of the placebo group ( $\mathrm{p}=0.03, \mathrm{p}=$ 0.32 versus placebo respectively). This suggests that vedolizumab may heal draining fistulae, but a study (ENTERPRISE, NCT 02630966) designed specifically to evaluate the effect has been concluded prematurely after 34 of a planned 100 patients were recruited (2018).

\section{Summary}

-Vedolizumab is safe and effective in CD and offers an appealing treatment option compared to anti-TNF therapy in the elderly, in those at risk of sepsis, or with a history of malignancy. The next anti-integrin likely to be licensed is etrolizumab, which thus far shows a similar safety and efficacy profile. 
Small molecule, S1P modulators could be the first oral biological agent available for the treatment of CD. Small molecules offer several advantages compared to monoclonal antibodies: linear pharmacokinetics, independent of pharmacodynamic factors, good tissue penetration and improved renal clearance with short half-lives. A short half-life is an advantage if an adverse event occurs. The availability of novel, oral IBD therapies would increase options for patients who prefer this route of administration, but currently necessitate daily therapy and some patients prefer less frequent parenteral therapy.

The last two decades have seen profound changes in the medical management of $\mathrm{CD}$ and the rate of change has accelerated such that within the next five years there may be several new drugs on the market. IBD physicians will have an armamentarium of available drugs, so choosing the appropriate biologic and deciding on precedence will remain a patient-centred decision until proteomic, metabolomic or other biomarkers are identified to guide choice and patientcentred outcome data are routinely reported.

\section{Practice Points:}

- Vedolizumab has led the way for leukocyte trafficking drugs to treat Crohn's disease, demonstrating an excellent safety profile and efficacy

- A slower onset of action may have to be traded off against a better safety profile and sustainability of effect than anti-TNF therapy

- Several other leukocyte trafficking agents are undergoing phase III trials, etrolizumab (inhibitor of $\alpha 4 \beta 7$ and $\alpha \mathrm{E} \beta 7$ ) and S1P modulators (etrasimod, ozanimod), with results expected in 2019-20.

\section{Research agenda:}

- Combination biological therapy with vedolizumab and anti-TNF or antiIL23 therapy

- Role of therapeutic drug monitoring for vedolizumab

- Impact of early anti-integrin therapy on the course of Crohn's disease

Acknowledgements: This work was supported by the National Institute for Health Research (NIHR) Oxford Biomedical Research Centre (BRC). The views expressed are those of the author(s) and not necessarily those of the NHS, the NIHR or the Department of Health.

\section{Role of the funding source: None}

\section{Conflicts of interest}

Sujata Biswas: None

Simon Travis: Grants/Research Support: AbbVie, Buhlmann, Celgene, IOIBD, Janssen, Lilly, Takeda, UCB, Vifor, and Norman Collisson Foundation. Consulting Fees: AbbVie, Allergan, Amgen, Arena, Asahi, Astellas, Biocare, Biogen, Boehringer Ingelheim, Bristol-Myers Squibb, Buhlmann, Celgene, Chemocentryx, Cosmo, Enterome, Ferring, Giuliani SpA, GSK, Genentech, Immunocore, Immunometabolism, Indigo, Janssen, Lexicon, Lilly, Merck, MSD, Netbiotix, Neovacs, Novartis, NovoNordisk, NPS Pharmaceuticals, Pfizer, Proximagen, 
Receptos, Roche, Shire, Sigmoid Pharma, SynDermix, Takeda, Theravance, Tillotts, Topivert, UCB, VHsquared, Vifor, Zeria. Speaker fees: AbbVie, Amgen, Biogen, Ferring, Janssen, Shire, Takeda. No stocks or share options.

RB: Grant/Travel/Research support: Takeda, Ferring. Speaking honoraria: AbbVie, Ferring, Janssen, Shire, Takeda. Consulting fees: Gilead, Emerge Health. 


\section{References}

1. Butcher EC, Scollay RG, Weissman IL. Organ specificity of lymphocyte migration: mediation by highly selective lymphocyte interaction with organspecific determinants on high endothelial venules. Eur J Immunol. 1980;10(7):556-61.

2. Cominelli F. Inhibition of leukocyte trafficking in inflammatory bowel disease. N Engl J Med. 2013;369(8):775-6.

3. Springer TA. Traffic signals for lymphocyte recirculation and leukocyte emigration: the multistep paradigm. Cell. 1994;76(2):301-14.

4. Rivera-Nieves J, Gorfu G, Ley K. Leukocyte adhesion molecules in animal models of inflammatory bowel disease. Inflamm Bowel Dis. 2008;14(12):171535 .

5. Yoshida N, Yamaguchi T, Nakagawa S, Nakamura Y, Naito Y, Yoshikawa T. Role of P-selectin and intercellular adhesion molecule-1 in TNB-induced colitis in rats. Digestion. 2001;63 Suppl 1:81-6.

6. Hamamoto N, Maemura K, Hirata I, Murano M, Sasaki S, Katsu K. Inhibition of dextran sulphate sodium (DSS)-induced colitis in mice by intracolonically administered antibodies against adhesion molecules (endothelial leucocyte adhesion molecule-1 (ELAM-1) or intercellular adhesion molecule-1 (ICAM-1)). Clin Exp Immunol. 1999;117(3):462-8.

7. Bendjelloul F, Maly P, Mandys V, Jirkovska M, Prokesova L, Tuckova L, et al. Intercellular adhesion molecule-1 (ICAM-1) deficiency protects mice against severe forms of experimentally induced colitis. Clin Exp Immunol. 2000;119(1):57-63.

8. Sans M, Panes J, Ardite E, Elizalde JI, Arce Y, Elena M, et al. VCAM-1 and ICAM-1 mediate leukocyte-endothelial cell adhesion in rat experimental colitis. Gastroenterology. 1999;116(4):874-83.

9. Burns RC, Rivera-Nieves J, Moskaluk CA, Matsumoto S, Cominelli F, Ley K. Antibody blockade of ICAM-1 and VCAM-1 ameliorates inflammation in the SAMP-1/Yit adoptive transfer model of Crohn's disease in mice. Gastroenterology. 2001;121(6):1428-36.

10. Podolsky DK, Lobb R, King N, Benjamin CD, Pepinsky B, Sehgal P, et al. Attenuation of colitis in the cotton-top tamarin by anti-alpha 4 integrin monoclonal antibody. J Clin Invest. 1993;92(1):372-80.

11. Kato S, Hokari R, Matsuzaki K, Iwai A, Kawaguchi A, Nagao S, et al. Amelioration of murine experimental colitis by inhibition of mucosal addressin cell adhesion molecule-1. J Pharmacol Exp Ther. 2000;295(1):183-9.

12. Yacyshyn B, Chey WY, Wedel MK, Yu RZ, Paul D, Chuang E. A randomized, double-masked, placebo-controlled study of alicaforsen, an antisense inhibitor of intercellular adhesion molecule 1, for the treatment of subjects with active Crohn's disease. Clin Gastroenterol Hepatol. 2007;5(2):215-20.

13. James DG, Seo DH, Chen J, Vemulapalli C, Stone CD. Efalizumab, a human monoclonal anti-CD11a antibody, in the treatment of moderate to severe Crohn's Disease: an open-label pilot study. Dig Dis Sci. 2011;56(6):1806-10.

14. Sandborn WJ, Lee SD, Tarabar D, Louis E, Klopocka M, Klaus J, et al. Phase II evaluation of anti-MAdCAM antibody PF-00547659 in the treatment of Crohn's disease: report of the OPERA study. Gut. 2018;67(10):1824-35. 
15. Targan SR, Feagan BG, Fedorak RN, Lashner BA, Panaccione R, Present $\mathrm{DH}$, et al. Natalizumab for the treatment of active Crohn's disease: results of the ENCORE Trial. Gastroenterology. 2007;132(5):1672-83.

16. Sandborn WJ, Colombel JF, Enns R, Feagan BG, Hanauer SB, Lawrance IC, et al. Natalizumab induction and maintenance therapy for Crohn's disease. $\mathrm{N}$ Engl J Med. 2005;353(18):1912-25.

17. Sandborn WJ, Feagan BG, Rutgeerts P, Hanauer S, Colombel JF, Sands BE, et al. Vedolizumab as induction and maintenance therapy for Crohn's disease. $\mathrm{N}$ Engl J Med. 2013;369(8):711-21.

18. Sandborn W, Cyrille M, Berner Hansen M, Feagan B, Loftus Jr E, Vermeire S, et al. OP035 Efficacy and safety of abrilumab (AMG 181/MEDI 7183) therapy for moderate to severe Crohn's disease. Journal of Crohn's and Colitis. 2017;11(suppl_1):S22-S3.

19. Keshav S, Vanasek T, Niv Y, Petryka R, Howaldt S, Bafutto M, et al. A randomized controlled trial of the efficacy and safety of CCX282-B, an orallyadministered blocker of chemokine receptor CCR9, for patients with Crohn's disease. PLoS One. 2013;8(3):e60094.

20. Feagan BG, Sandborn WJ, D'Haens G, Lee SD, Allez M, Fedorak RN, et al. Randomised clinical trial: vercirnon, an oral CCR9 antagonist, vs. placebo as induction therapy in active Crohn's disease. Aliment Pharmacol Ther. 2015;42(10):1170-81.

21. Malizia G, Calabrese A, Cottone M, Raimondo M, Trejdosiewicz LK, Smart CJ, et al. Expression of leukocyte adhesion molecules by mucosal mononuclear phagocytes in inflammatory bowel disease. Gastroenterology. 1991;100(1):1509.

22. Briskin M, Winsor-Hines D, Shyjan A, Cochran N, Bloom S, Wilson J, et al. Human mucosal addressin cell adhesion molecule-1 is preferentially expressed in intestinal tract and associated lymphoid tissue. The American journal of pathology. 1997;151(1):97.

23. Pullen N, Molloy E, Carter D, Syntin P, Clemo F, Finco-Kent D, et al. Pharmacological characterization of PF-00547659, an anti-human MAdCAM monoclonal antibody. Br J Pharmacol. 2009;157(2):281-93.

24. Vermeire S, Sandborn WJ, Danese S, Hebuterne X, Salzberg BA, Klopocka $M$, et al. Anti-MAdCAM antibody (PF-00547659) for ulcerative colitis (TURANDOT): a phase 2, randomised, double-blind, placebo-controlled trial. Lancet. 2017;390(10090):135-44.

25. Ghosh S, Goldin E, Gordon FH, Malchow HA, Rask-Madsen J, Rutgeerts P, et al. Natalizumab for active Crohn's disease. N Engl J Med. 2003;348(1):24-32.

26. Sandborn W, Cyrille M, Hansen M, Feagan B, Loftus Jr E, Rogler G, et al. OP034 Efficacy and safety of abrilumab in subjects with moderate to severe ulcerative colitis: results of a phase $2 \mathrm{~b}$, randomised, double-blind, multiple-dose, placebo-controlled study. Journal of Crohn's and Colitis. 2017;11(suppl_1):S21S2.

27. Vermeire S, O'Byrne S, Keir M, Williams M, Lu TT, Mansfield JC, et al. Etrolizumab as induction therapy for ulcerative colitis: a randomised, controlled, phase 2 trial. Lancet. 2014;384(9940):309-18.

28. William S, Julian P, Jennifer J, Azra H, Rhian J, Zaineb S, et al. etrolizumab as Induction Therapy in Moderate to Severe Crohn's Disease: Results From Bergamot Cohort 1: p-011. American Journal of Gastroenterology. 2018;113:S3. 
29. Pelletier D, Hafler DA. Fingolimod for multiple sclerosis. N Engl J Med. 2012;366(4):339-47.

30. Deguchi Y, Andoh A, Yagi Y, Bamba S, Inatomi O, Tsujikawa T, et al. The S1P receptor modulator FTY720 prevents the development of experimental colitis in mice. Oncol Rep. 2006;16(4):699-703.

31. Sandborn WJ, Feagan BG, Wolf DC, D'Haens G, Vermeire S, Hanauer SB, et al. Ozanimod Induction and Maintenance Treatment for Ulcerative Colitis. N Engl J Med. 2016;374(18):1754-62.

32. Zhu L, Gibson P, Currle DS, Tong Y, Richardson RJ, Bayazitov IT, et al. Prominin 1 marks intestinal stem cells that are susceptible to neoplastic transformation. Nature. 2009;457(7229):603-7.

33. Bye WA, Jairath V, Travis SPL. Systematic review: the safety of vedolizumab for the treatment of inflammatory bowel disease. Aliment Pharmacol Ther. 2017;46(1):3-15.

34. Liu J, Sylwestrzak G, Ruggieri AP, DeVries A. Intravenous Versus Subcutaneous Anti-TNF-Alpha Agents for Crohn's Disease: A Comparison of Effectiveness and Safety. J Manag Care Spec Pharm. 2015;21(7):559-66.

35. Bryant RV, Sandborn WJ, Travis SP. Introducing vedolizumab to clinical practice: who, when, and how? J Crohns Colitis. 2015;9(4):356-66.

36. Feagan BG, Bhayat F, Khalid M, Blake A, Travis SPL. Respiratory Tract Infections in Patients With Inflammatory Bowel Disease: Safety Analyses From Vedolizumab Clinical Trials. J Crohns Colitis. 2018;12(8):905-19.

37. Colombel JF, Sands BE, Rutgeerts P, Sandborn W, Danese S, D'Haens G, et al. The safety of vedolizumab for ulcerative colitis and Crohn's disease. Gut. 2017;66(5):839-51.

38. Kotlyar DS, Lewis JD, Beaugerie L, Tierney A, Brensinger CM, Gisbert JP, et al. Risk of lymphoma in patients with inflammatory bowel disease treated with azathioprine and 6-mercaptopurine: a meta-analysis. Clin Gastroenterol Hepatol. 2015;13(5):847-58 e4; quiz e48-50.

39. Long MD, Martin CF, Pipkin CA, Herfarth HH, Sandler RS, Kappelman MD. Risk of melanoma and nonmelanoma skin cancer among patients with inflammatory bowel disease. Gastroenterology. 2012;143(2):390-9 e1.

40. McKenna MR, Stobaugh DJ, Deepak P. Melanoma and non-melanoma skin cancer in inflammatory bowel disease patients following tumor necrosis factoralpha inhibitor monotherapy and in combination with thiopurines: analysis of the Food and Drug Administration Adverse Event Reporting System. J Gastrointestin Liver Dis. 2014;23(3):267-71.

41. Peyrin-Biroulet L, Khosrotehrani K, Carrat F, Bouvier AM, Chevaux JB, Simon $\mathrm{T}$, et al. Increased risk for nonmelanoma skin cancers in patients who receive thiopurines for inflammatory bowel disease. Gastroenterology. 2011;141(5):1621-28 e1-5.

42. Vermeire S, Loftus EV, Jr., Colombel JF, Feagan BG, Sandborn WJ, Sands BE, et al. Long-term Efficacy of Vedolizumab for Crohn's Disease. J Crohns Colitis. 2017;11(4):412-24.

43. Bernstein CN, Blanchard JF, Kliewer E, Wajda A. Cancer risk in patients with inflammatory bowel disease: a population-based study. Cancer. 2001;91(4):854-62.

44. Sands BE, Feagan BG, Rutgeerts P, Colombel JF, Sandborn WJ, Sy R, et al. Effects of vedolizumab induction therapy for patients with Crohn's disease in 
whom tumor necrosis factor antagonist treatment failed. Gastroenterology. 2014;147(3):618-27 e3.

45. Hanzel J, Sever N, Ferkolj I, Stabuc B, Smrekar N, Kozelj M, et al. Mo1836 Vedolizumab Trough Levels at Week 6 Predict Endoscopic and Clinical Remission in Inflammatory Bowel Disease. Gastroenterology. 2018;154(6):S819.

46. Rosario M, Wyant T, Leach T, Sankoh S, Scholz C, Parikh A, et al. Vedolizumab Pharmacokinetics, Pharmacodynamics, Safety, and Tolerability Following Administration of a Single, Ascending, Intravenous Dose to Healthy Volunteers. Clin Drug Investig. 2016;36(11):913-23.

47. Charpentier C, Salleron J, Savoye G, Fumery M, Merle V, Laberenne JE, et al. Natural history of elderly-onset inflammatory bowel disease: a populationbased cohort study. Gut. 2014;63(3):423-32.

48. Navaneethan U, kumar Kommaraju K, Edminster T, Zhu X, Wilson K, Glover SC. Mo1904 efficacy and safety of vedolizumab in elderly patients with inflammatory bowel disease. Gastroenterology. 2016;150(4):S812.

49. Mahadevan U, Vermeire S, Lasch K, Abhyankar B, Bhayat F, Blake A, et al. Vedolizumab exposure in pregnancy: outcomes from clinical studies in inflammatory bowel disease. Aliment Pharmacol Ther. 2017;45(7):941-50.

50. Lightner AL, Raffals LE, Mathis KL, Cima RR, Tse CS, Pemberton JH, et al. Postoperative outcomes in vedolizumab-treated patients undergoing abdominal operations for inflammatory bowel disease. Journal of Crohn's and Colitis. 2017;11(2):185-90.

51. Stringfield S, Parry L, Sandborn W, Ramamoorthy S, Eisenstein S, editors. PATIENTS ON VEDOLIZUMAB HAVE A HIGH RATE OF POSTOPERATIVE COMPLICATIONS. DISEASES OF THE COLON \& RECTUM; 2016: LIPPINCOTT WILLIAMS \& WILKINS TWO COMMERCE SQ, 2001 MARKET ST, PHILADELPHIA ....

52. Koh S, Zaghiyan K, Fleshner P, editors. Safety and efficacy of the perioperative use of vedolizumab in medically refractory IBD patients. Does" gut-specificity" impact surgical morbidity? DISEASES OF THE COLON \& RECTUM; 2016: LIPPINCOTT WILLIAMS \& WILKINS TWO COMMERCE SQ, 2001 MARKET ST, PHILADELPHIA ....

53. Kopylov U, Ben-Horin S, Zmora 0, Eliakim R, Katz LH. Anti-tumor necrosis factor and postoperative complications in Crohn's disease: systematic review and meta-analysis. Inflamm Bowel Dis. 2012;18(12):2404-13.

54. Tadbiri S, Peyrin-Biroulet L, Serrero M, Filippi J, Pariente B, Roblin X, et al. Impact of vedolizumab therapy on extra-intestinal manifestations in patients with inflammatory bowel disease: a multicentre cohort study nested in the OBSERV-IBD cohort. Aliment Pharmacol Ther. 2018;47(4):485-93.

55. Dubinsky MC, Cross RK, Sandborn WJ, Long M, Song X, Shi N, et al. Extraintestinal Manifestations in Vedolizumab and Anti-TNF-Treated Patients With Inflammatory Bowel Disease. Inflamm Bowel Dis. 2018. 